\title{
Development of Board Placing Robot
}

\author{
Hiroshi Yoshinada*, Toshiyuki Sawano*, Ikukazu Takahashi*, Hidetaka Suzuki* \\ Eiji Muro**, Haruo Hosino** \\ Jiro Hakamazuka***, Minoru Iichi**** \\ * $\quad$ KOMATSU Ltd., RESEARCH DIVISION, \\ 1200, Manda, Hiratsuka-shi, Kanagawa, 254, JAPAN \\ ** TAKENAKA RESEARCH \& DEVELOPMENT INSTITUTE, \\ 5, 1-chome, Ohtsuka, Inzai-machi, Inba-gun, Chiba, 270-13, JAPAN \\ *** JAPAN INTERIOR CONSTRUCTORS ASSOCIATION, \\ 1-5-10, Nihonbashi Ningyocho, Chuou-ku, Tokyo, 103, JAPAN \\ **** JAPAN GYPSUM BOARD ASSOCIATION, \\ 2-13-12, Nishi-Shinbashi, Minato-ku, Tokyo, 105, JAPAN
}

\begin{abstract}
The development of the board placing robot for interior finishing works is described. The featured concept is entirely new and the structure of the system is almost different from the former board installation robots. The robot is developed as a personal use one for a board installation worker and the robot coexists with a worker in the same work place. To obtain the security between the robot and worker, the compliant pneumatic actuator system has been applied. The robot is designed compactly and also a new telescopic configuration can cover wide work area from a wall to ceiling. The simplified structure of the system can cut the time of preparation and realize to put it into practical use.

In this paper the structure of the system and the results of the practical tests are described.
\end{abstract}

KEYWORDS ; Interior finishing work, Board installation, Man-Robot coexistence, Pneumatic actuator, 


\section{INTRODUCTION}

In the interior finishing works, installation of wall and ceiling boards is very hard work for the labors because it forces them unusual posture and requires large muscular power for many hours. Furthermore to improve a fireproof ability and sound insulation, there is a tendency for thicker gypsum boards to be used and a work has become much harder. Therefore the increase of advanced aged labors and lack of skilled workers have been serious problem. Until today several kinds of robots have been developed which aim the automatic or semi-automatic installation of a wall and ceiling board. However they are not used popularly, because most of them are too large and heavy to put into practical use and board sizes or shapes are strictly restricted. Of course they were not effective from an economical view point.

From 1994, authors have been developing a board placing robot with new concept. This robot is developed as a personal use one for a board install worker and it is considered a kind of human muscular power assist equipment. Shortly, the robot will do tasks need muscular power such as holding and supporting a board and the worker will do tasks need skills such as adjusting position and fixing a board. To achieve a security between a robot and worker, a safer pneumatic actuator system is used for a robot. And simplified manmachine interface could eliminate a time to master operation.

The developed robot is very compact and light weight. It can be applied to an interior finishing work in a small room. And this robot is almost free from the restriction of board sizes and shapes and the simplified system structure can cut a time for preparation.

Below authors present the system structures and the results of the practical tests in detail.

\section{BASIC CONCEPTS}

An installation work of interior finishing boards is divided in following six tasks.

(Task-1) Carry a board from a stock.

(Task-2) Measure actual dimensions of furring flames.

(Task-3) Draw cutting lines on a board and cut it if needed.

(Task-4) Handle and support a board on furring flames.

(Task-5) Adjust a position and install a board to put out a gap between a next board.

(Task-6) Fix a board with screws. 
In a conventional method, a worker repeats Tasks from 1 to 6 successively. In the case of a robotized system, it is easily expected to be difficult and noneffective to accomplish all these tasks automatically by the robot. In these six tasks, Task-1 and Task-4 require a large muscular power and repeating them is very hard for a worker. On the other hand, Task-2, Task-3, Task- 5 and Task- 6 need skillful techniques of a worker, but these tasks are not so hard physically. Considering these points and problems of previous exampies, following basic concepts were drafted.

(Concept-1) Tasks that need skills such as Task-2,3,5,6 are accomplished by a worker.

(Concept-2) Robot behaves itself as a kind of muscular power assist equipment.

(Concept-3) Man-Robot coexistence and simplified the man-robot interface.

(Concept-4) Applicability for a small room finishing work.

(Concept-5) Carrying easily with a hand (or both hands).

According to these concepts, the structure of the robot system were investigated in detail.

\section{MECHANICAL DESIGN}

For the realization of Concept-5,6 in Chapter 2, the robot must be designed as compactly as possible. On the other hand, to perform an installation work on a high ceiling, a robot must have a wide work area. In order to satisfy these opposed conditions, various kinds of configurations have been investigated and a configuration shown in Figure 1 was made up finally. This configuration has a small wheeled push car as a base stand and a telescopic actuator is mounted vertically on this push car. On the end of a telescopic actuator, a horizontal multi joint manipulator is attached. This manipulator has five degrees of freedom and its configuration is almost similar to an industrial SCARA robot.

A location of the robot is decided roughly using a push car. And a location of a board is decided precisely using a telescopic actuator and a manipulator. A vertical telescopic cylinder enables the robot to cover the wide work area from a wall to a ceiling.

Figure 2 shows a side view of a developed robot. All actuators of the robot are driven by pneumatic actuators. The reason why will be mentioned later. A pneumatic telescopic cylinder covers a wide vertical work area and it extends from a height of $875 \mathrm{~mm}$ to $2750 \mathrm{~mm}$. A horizontal manipulator is driven using pneumatic rotary actuators. These 
actuators are controlled using nozzle-flapper type pneumatic servo valves. The robot is connected to a $0.75 \mathrm{kw}$ air compressor. As a board catcher, a vacuum cup is attached on the end of the manipulator. As a negative pressure is produced using Venturi nozzle, a vacuum cup is also connected to the air compressor through a solenoid valve. Compressed pressure is regulated about $0.5 \mathrm{MPa}$.

A base push car consists of four outriggers and a caster wheel is attached on the end of each outrigger. These outriggers can be folded compactly. The weight of a robot complete was about $50 \mathrm{~kg}$. The robot is easily divided into three pieces and each piece is light enough to carry with a hand. A rated payload of the robot is $20 \mathrm{~kg}$ and this value meets to the weight of a large gypsum board commonly used. The general view of the robot is shown in Figure 3.

\section{CONTROL SYSTEM DESIGN}

\subsection{Pneumatic Actuator System}

In order to realize man-robot coexistence, it must be considered at first to eliminate a danger of a robot that will injure a worker. Of course, the protection for the system failure is important. Moreover it is indispensable that the actuator system possesses safe characteristics such as adequate compliance and moderate response naturally. To achieve this purpose, a pneumatic actuator system is considered to be superior to the other types, because the compressibility of air can provide these characteristics naturally.

On the other hand, compressibility of air will cause vibration of the system and it becomes difficult to control the robot precisely. Especially regarding a pneumatic rotary actuator, due to a large static friction force which is mainly produced by a rotary vane seal, it involves great difficulties in control.

Figure 4 shows a control schema of a pneumatic rotary actuator. Pressure feedback gain $\boldsymbol{K}_{\boldsymbol{p}}$ can control the vibration of the system caused by the compressibility of air and angular velocity feedback gain $\boldsymbol{K}_{v}$ can inhibit a stick-slip phenomena caused by a friction of actuator seals. Regarding both static and dynamic characteristics of the servo system, good results have been obtained to adjust the values of $\boldsymbol{K}_{\boldsymbol{p}}$ and $\boldsymbol{K}_{v}$ properly. Joint compliance is about $33 \mathrm{Nm} / \mathrm{rad}$ and a band width of frequency response is adjusted to be about $1.5 \mathrm{~Hz}$. Although these values are so soft and slow compared to an usual servo system, they are considered to be adequate values to eliminate a danger from a man-robot coexistence system. 


\subsection{Control system and Man-Robot Interface}

Generally the environment of construction is not clean and unstable compared to industries. For a practical application of a robot in construction environment, the simplicity and reliability of a system are more important. In order to achieve this object, several kinds of robot controllers have been examined. Finally a small controller for an unmanned dump truck has been chosen and some modifications have been made to use it as a robot controller. The controller is packaged in a heavy-duty metal box and it contains processors, memories, several kinds of input/output devices, valve drive amplifiers, filters and voltage regulators. Although processing ability is somewhat inferior to an ordinary robot controller, this defect could be overcome by some programming techniques. This controller is small enough to equip on the robot and also it can endure a bad environment.

In the case that the robot behaves itself as a muscular power assist equipment, it is very important that the robot assists purely a muscular load and it does not obstruct the worker's skills and know-hows. For this reason, an ordinary robot operation box which has many switches and levers is not suitable at all. An intuitive operation system is required for this kind of robot. In order to achieve this object, authors have developed a hybrid operation system using below two operation modes.

(MODE-1) Fukuda's group proposed a concept of a manipulator in construction based on man-robot cooperation work.[1] Their mono-levered direct teaching system is considered to be one of the best solutions for this purpose. As first operation mode, we have used a similar system, but simplified it to omit needless functions for our robot. Using the joystick levers attached on the end of a forearm, a worker can easily control the direction and speed of the movement of the manipulator. This first operation mode is fitted to the rough positioning of a board such as Task-1,4 in Chapter 2 .

(MODE-2) In addition to MODE-1, authors have developed a more intuitive operation system. In this second mode, operator can move the manipulator freely to push and pull any parts of the manipulator without switches or levers. In this mode, the original position control servo system of the manipulator is changed to the force control one and a proper input value which cancels a gravity and inertia and friction force is input to the servo system. This second operation mode is fitted to the fine positioning such as Task-5.

A conversion one mode to the other is very simple. It does not need any switches 
operation. According to the workers natural action, the mode is converted unconsciously. The following principle has been applied to this conversion system.

In MODE-1, an electric current of the valve drive amplifier is in proportion to the displacement of the joystick lever. In the case that the lever is in a neutral position, each joint of the manipulator is servo-locked and the electrical current of the amplifier is kept constant. Remaining in this state, if the worker applies a force on the manipulator, the electrical current rises to keep the original position. Setting an adequate threshold value, if the electrical current exceeds this value, MODE-1 is converted into MODE-2. When a worker touch a joystick lever again, MODE-2 is returned to MODE-1.

A schema of the control system is shown in Figure 5.

\section{RESULTS OF THE PRACTICAL TESTS}

The practical test has been done in a condominium room finishing work. A height of a ceiling is $2700 \mathrm{~mm}$, a length of a floor is $3600 \times 3600 \mathrm{~mm}$. A gypsum board $1820 \times$ $910 \mathrm{~mm}$ and $9.5 \mathrm{~mm}$ thick was used for a wall and ceiling finishing. Before the test, a worker was trained for the operation in about one hour. In regard to both cases of a new method using a robot and a conventional method without robot, the time required for the installation was measured. The results are shown in Table 1.

Table 1 Test Results

\begin{tabular}{ccc}
\hline Cycle Time & New Method with Robot & Conventional Method \\
\hline Ceiling Board & $186 \mathrm{sec}$. & $438 \mathrm{sec}$. \\
Wall Board & $281 \mathrm{sec}$. & $345 \mathrm{sec}$. \\
\hline
\end{tabular}

The cycle time in Table 1 indicates a total time per a board accomplishing six tasks in Chapter 2. In both cases of a ceiling and wall, sufficient advantages were obtained with a robotized new method. And alternative test using a larger gypsum board $910 \times 2430 \mathrm{~mm}$ and $12.5 \mathrm{~mm}$ thick shows that the cycle time does not become worse in the case of the robotized new method. These results suggests that the developed robot can reduce a physical fatigue of a worker and improve the efficiency of the installation work. The actual scene of the practical test is shown in Figure 6. 


\section{CONCLUSION}

The board placing robot which assists a worker in interior finishing works was described. Through the practical tests, the validity of the new concepts and new configuration of the robot has been confirmed. Refining and commercializing are one of the major targets in the next stage. Also finding the alternative applications in the other fields is our greatest concern.

\section{ACKNOWLEDGMENTS}

The authors are grateful to SUKETOMO CORPORATION for the helpful advises and assistance for the practical test.

This work was performed under the management of JAPAN SMALL BUSINESS CORPORATION (J.S.) as a part of the technological development project sponsored by J.S..

\section{REFERENCE}

[1]Fukuda, T. et al, "A NEW ROBOTIC MANIPULATOR IN CONSTRUCTION BASED ON MAN-ROBOT COOPERATION WORK",

8th. International Symposium on Automatic and Robotics in Construction, Proceedings, Volume 1, pp.239-245, 1991 


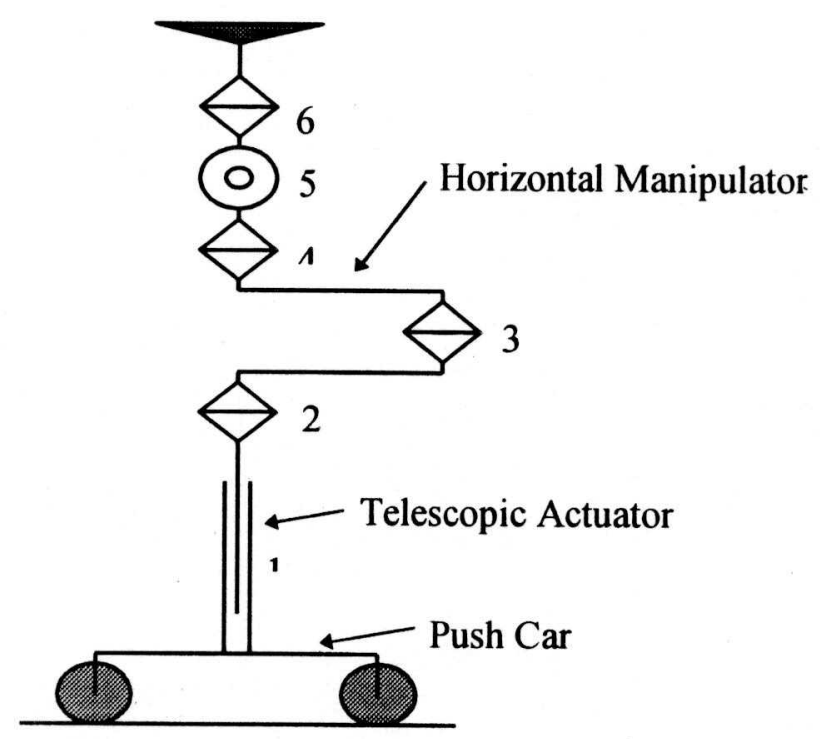

Figure 1 Configuration of the Robot

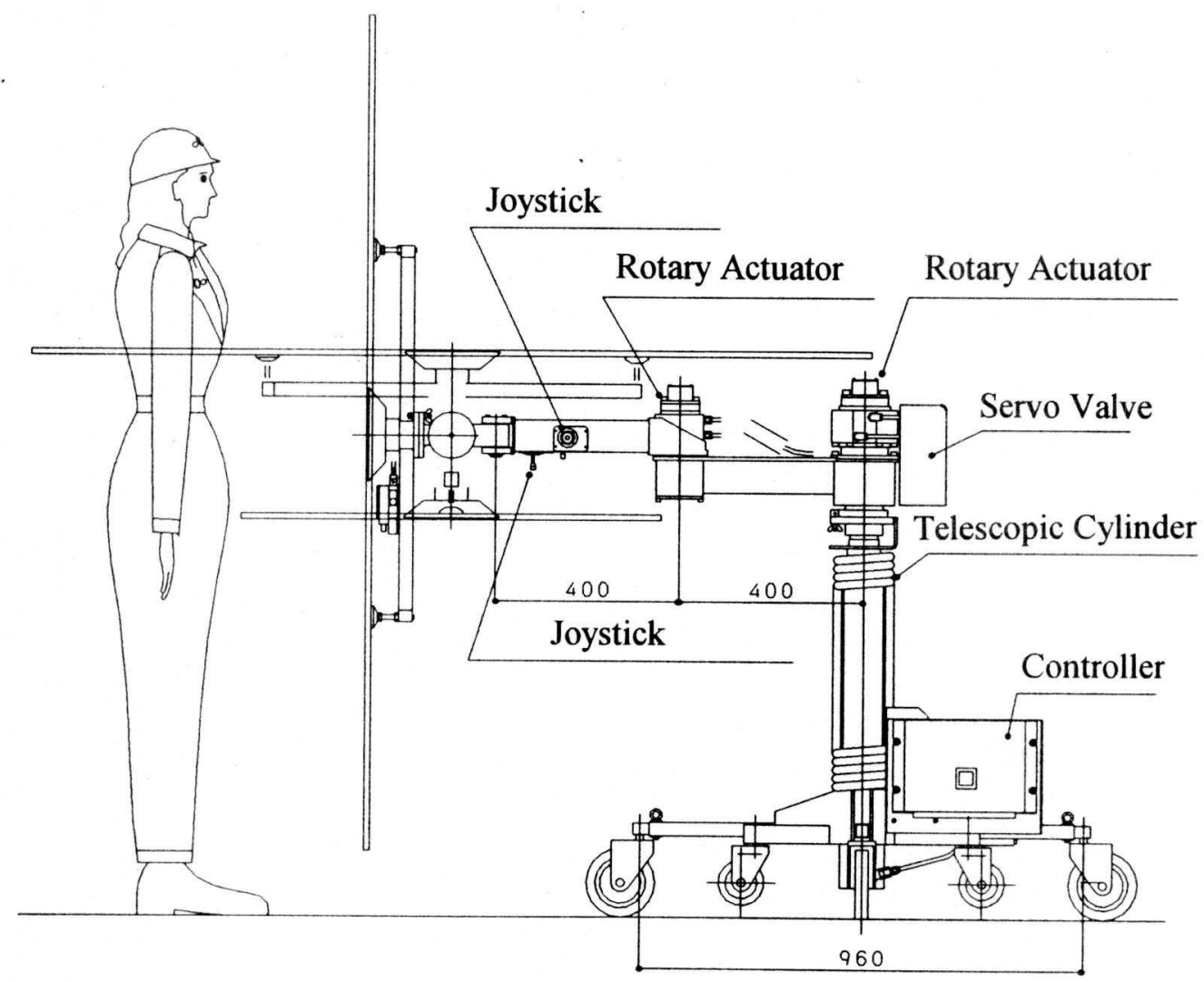

Figure 2 Side View of the Robot 


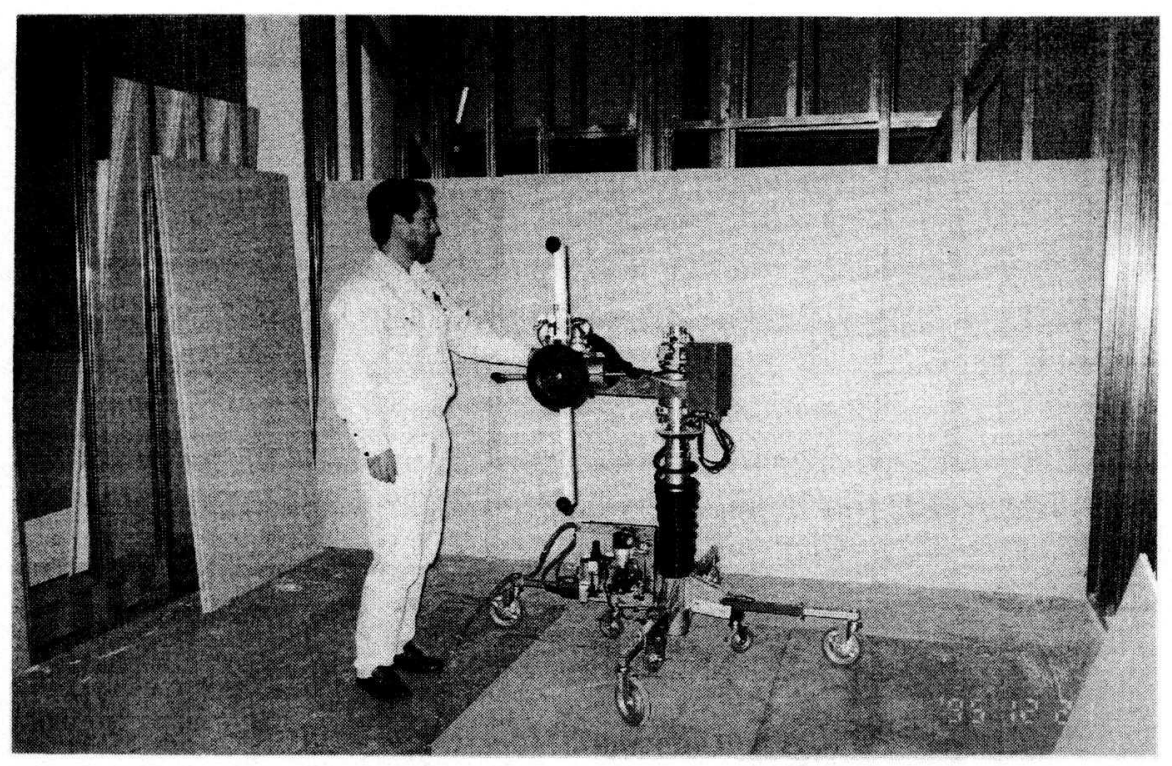

Figure 3 General View of the Robot

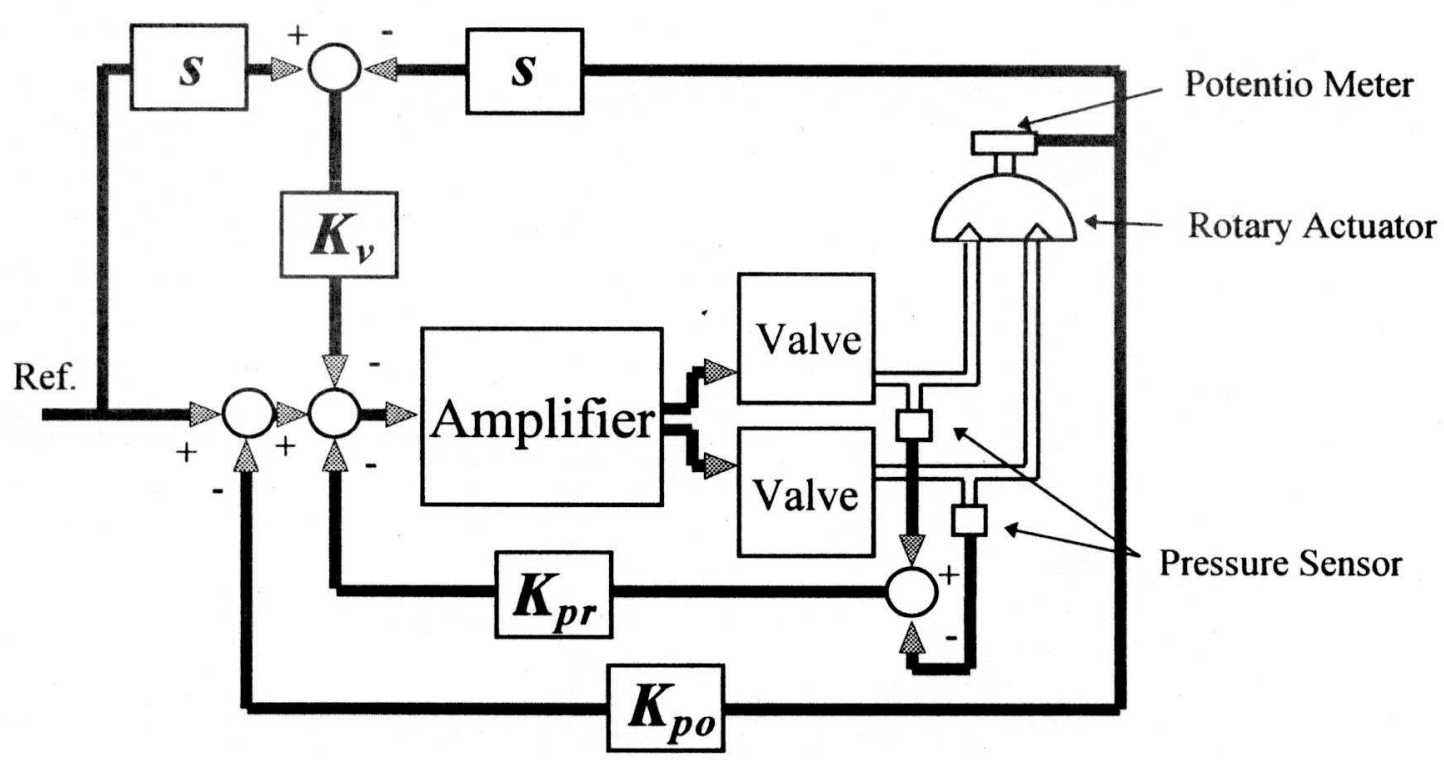

Figure 4 Control Schema of Pneumatic Rotary Actuator 


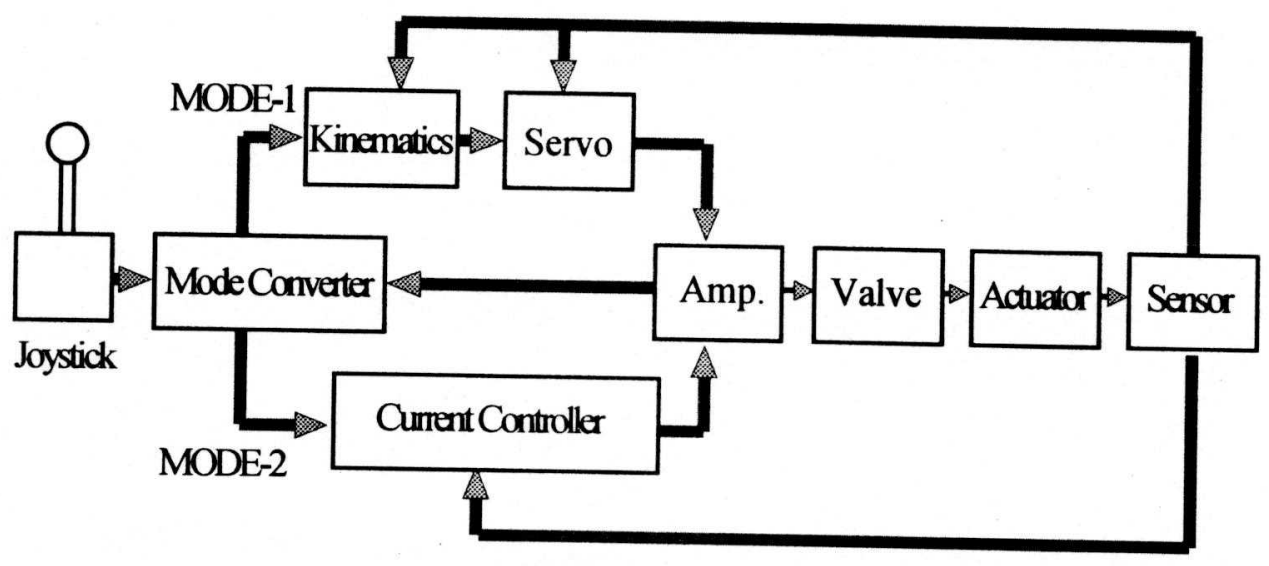

Figure 5 Schema of Control System

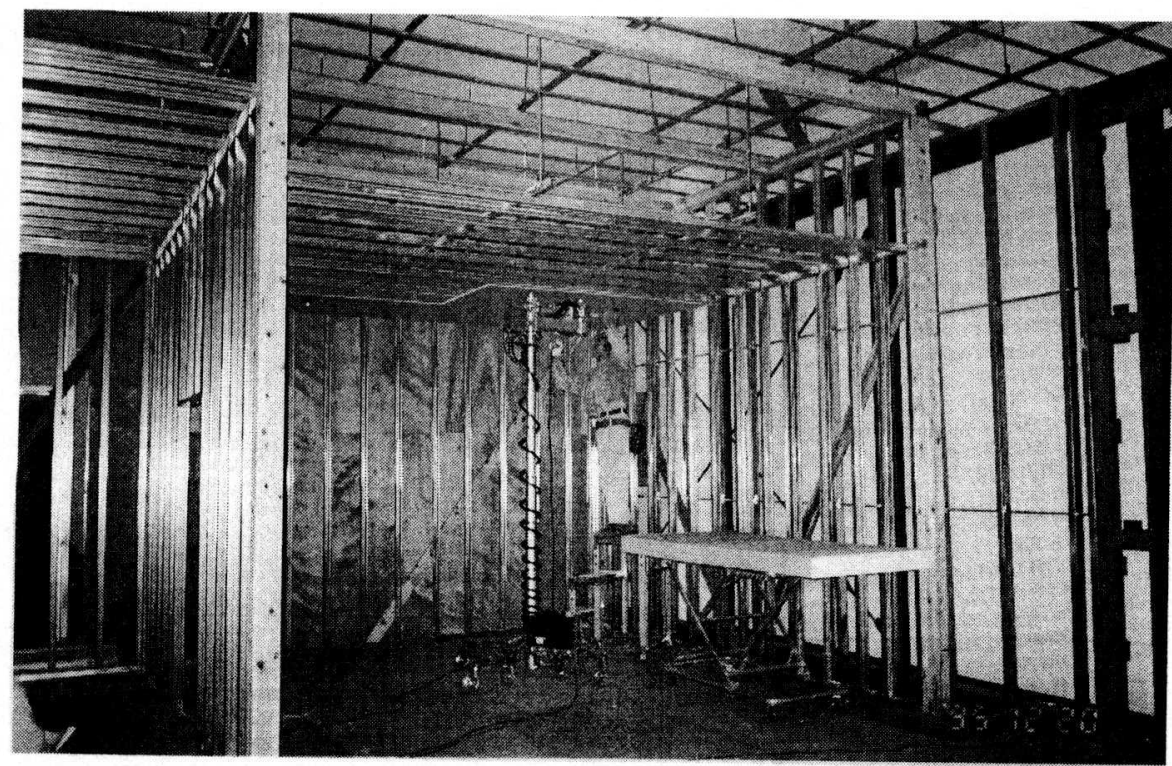

Figure 6 Practical Test . 